CERN-TH/2003-277

TTP03-36

November 2003

\title{
The analytic value of a 4-loop sunrise graph in a particular kinematical configuration.
}

\author{
S. Laporta,,$^{a, b, 1}$ P. Mastrolia ${ }^{c, d, 2}$ and E. Remiddi ${ }^{e, c, a, 3}$ \\ ${ }^{a}$ INFN, Sezione di Bologna, I-40126 Bologna, Italy \\ ${ }^{b}$ Dipartimento di Fisica, Università di Parma, I-43100 Parma, Italy \\ ${ }^{c}$ Dipartimento di Fisica, Università di Bologna, I-40126 Bologna, Italy \\ ${ }^{d}$ Institut für Theoretische Teilchenphysik, Universität Karlsruhe, D-76128 Karlsruhe, Germany \\ e Theory Division, CERN, CH-1211 Geneva 23, Switzerland
}

\begin{abstract}
The 4-loop sunrise graph with two massless lines, two lines of equal mass $M$ and a line of mass $m$, for external invariant timelike and equal to $m^{2}$ is considered. We write differential equations in $x=m / M$ for the Master Integrals of the problem, which we Laurent-expand in the regularizing continuous dimension $d$ around $d=4$, and then solve exactly in $x$ up to order $(d-4)^{3}$ included; the result is expressed in terms of Harmonic PolyLogarithms of argument $x$ and maximum weight 7 . As a by product, we obtain the $x=1$ value, expected to be relevant in QED 4-loop static quantities like the electron $(g-2)$. The analytic results were checked by an independent precise numerical calculation.
\end{abstract}

Key words: Feynman diagrams, Multi-loop calculations

PACS: 11.15.Bt, 12.20.Ds

\footnotetext{
${ }^{1}$ e-mail: Stefano.Laporta@bo.infn.it

e-mail: Pierpaolo.Mastrolia@bo.infn.it

3 e-mail: Ettore.Remiddi@bo.infn.it
} 


\section{Introduction}

This paper is devoted to the analytic evaluation of the Master Integrals (MI's) associated to the 4-loop sunrise graph with two massless lines, two massive lines of equal mass $M$, another massive line of mass $m$, with $m \neq M$, and the external invariant timelike and equal to $m^{2}$, as depicted in Fig. [

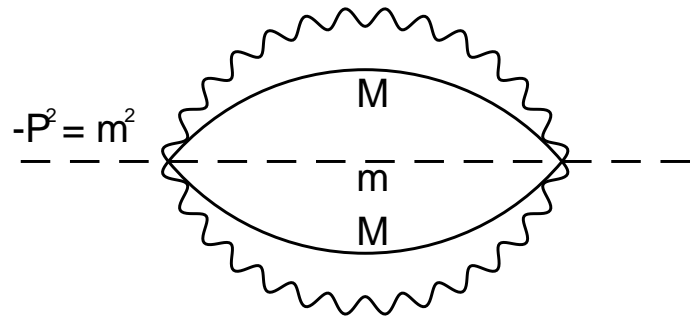

Figure 1: The considered 4-loop sunrise graph.

We will follow for the analytic integration the differential equation method already proposed in [1], further developped in [2] (and then used in [3], [4] and in many subsequent applications which would be too long to report here), as well as the finite difference method [5], [6] for an independent numerical check.

The differential equation method was already followed in two similar two- and three-loop calculations [7], [8]; the fact that its use could be extended without major changes to the present four-loop calculation witnesses for its generality and power. Among the advantages of the method, it allows a rather clear separation of the merely algebraic part of the work (which is, not surprisingly, always very heavy in this kind of multiloop calculations, and can be most conveniently processed by a computer algebra program, in our case FORM [9]), from the really analytic issues of the problem, which can then be better investigated without the disturbance of the algebraic complexity. In our case, indeed, the heart of the analytic calculation was the study of a homogeneous fourth order differential equation, whose solutions turned out to be, in a remarkably simple way, either a rational fraction or repeated quadratures of rational fractions. The required four-loop integral could then be obtained almost mechanically by repeated quadratures in terms of Harmonic PolyLogarithms [10].

Several other different approaches to the analytic evaluation of multiloop integrals are available in the literature, such as the powerful asymptotic expansion method (a fairly complete account can be found in the recent book [11]), and it would be interesting to compare the advantages and drawbacks of the various methods for the exacting, four-loop integration which we consider; but as the results of the present paper are new, a meaningful comparison cannot yet be carried out.

Following, as already said above, the approach already used in [7] and [8], we identify the MI's of the current problem within the continuous $d$-dimensional regularization, write the system of differential equations in $x=m / M$ satisfied by the MI's, convert it into a higher order differential equation for a single MI, Laurent-expand in $(d-4)$ around $d=4$, solve the associated homogeneous equation at $d=4$ (as in previous cases, the solutions of the homogeneous equation are surprisingly simple) and then use recursively Euler's method of the variation of the constants for obtaining the coefficient of the $(d-4)$ expansion in closed analytic form. The result involves Harmonic PolyLogarithms (HPL's) [10] of argument $x$ and weight increasing with the order in $(d-4)$. We push the analytic calculation, which works up to virtually any order in $(d-4)$, up to $(d-4)^{3}$ included, involving HPL's of weight up to $w=7$ included. The integration constants are fixed at $x=0$; as a by product we obtain the values at $x=1$, which are relevant in the evaluation of 4-loop static quantities such as the electron $(g-2)$ in QED. The result was checked and confirmed by an independent numerical calculation performed with the method of [5], [6].

The plan of the paper is the following. In Section 2 we define the Master Integrals and write the 
differential equations; in Section 3 we study the $x \rightarrow 0$ behaviour; in Section 4 we work out the Laurentexpansion in $(d-4)$ and discuss the associated homogeneous equation; in Section 5 we write down the solutions by Euler's method of the variation of the constants and give the $x=1$ values; Section 6 deals with the independent numerical calculation by which we check the results at $x=1$ of the previous Section. in Section 7 we carry out the evaluation of the $x \rightarrow 0$ values by direct integration in the parametric space.

\section{The Master Integrals and the Differential Equations}

We find that the problem has 5 MI's, which we choose to be

$$
F_{i}\left(d, M^{2}, m^{2}, P^{2}=-m^{2}\right)=\frac{1}{(2 \pi)^{4(d-2)}} \int \frac{d^{d} k_{1} d^{d} k_{2} d^{d} k_{3} d^{d} k_{4} N_{i}}{k_{1}^{2} k_{2}^{2}\left(k_{3}^{2}+M^{2}\right)\left(k_{4}^{2}+M^{2}\right)\left[\left(P-k_{1}-k_{2}-k_{3}-k_{4}\right)^{2}+m^{2}\right]},
$$

where the 5 numerators $N_{i}$ are $\left(M^{2}, k_{1} \cdot k_{3}, p \cdot k_{3}, k_{1} \cdot k_{2}, p \cdot k_{2}\right)$. In terms of the dimensionless variable $x=m / M$ and putting $P=M p$ one can introduce 5 dimensionless functions $\Phi_{i}(d, x)$ through

$$
F_{i}\left(d, M^{2}, m^{2}, P^{2}=-m^{2}\right)=M^{4 d-8} C^{4}(d) \Phi_{i}(d, x)
$$

where $C(d)=(4 \pi)^{\frac{4-d}{2}} \Gamma(3-d / 2)$ is an overall loop normalization factor, with the limiting value $C(4)=1$ at $d=4$. Some of the formulae which will follow (in particular the differential equations) are slightly simpler when written in terms of $x^{2}$ rather than $x$; but as $x$ is the most convenient variable for expressing the final analytic results, we stick to $x$ from the very beginning.

As in [7] [8], the derivation of the system of differential equations is straightforward; the derivatives of the MI's, i.e. of the 5 functions $\Phi_{i}(d, x)$, with respect to $x$ are easily carried out in their representation as loop-integrals Eq.(2.1); when the result is in turn expressed in terms of the same MI's, one obtains the following linear system of first order differential equations in $x$

$$
\begin{aligned}
\frac{d \Phi_{1}(d, x)}{d x}= & \left\{\frac{(3 d-7)}{x}+\frac{3(d-2)}{2(1-x)}-\frac{3(d-2)}{2(1+x)}\right\} \Phi_{1}(d, x)-\left\{\frac{3(d-2)}{x}+\frac{3(d-2)}{2(1-x)}\right. \\
& \left.-\frac{3(d-2)}{2(1+x)}\right\}\left(3 \Phi_{2}(d, x)-3 \Phi_{3}(d, x)+\Phi_{4}(d, x)-3 \Phi_{5}(d, x)\right), \\
\frac{d \Phi_{2}(d, x)}{d x}= & -\frac{(d-2)}{x}\left(\Phi_{2}(d, x)-2 \Phi_{3}(d, x)+\Phi_{4}(d, x)-2 \Phi_{5}(d, x)\right), \\
\frac{d \Phi_{3}(d, x)}{d x}= & -\left\{\frac{3(d-2)}{2(1-x)}-\frac{3(d-2)}{2(1+x)}\right\}\left(\Phi_{1}(d, x)-3 \Phi_{2}(d, x)-\Phi_{4}(d, x)\right) \\
& -\left\{\frac{3(d-2)}{x}+\frac{9(d-2)}{2(1-x)}-\frac{9(d-2)}{2(1+x)}\right\}\left(\Phi_{3}(d, x)+\Phi_{5}(d, x)\right), \\
\frac{d \Phi_{4}(d, x)}{d x}= & \frac{2(d-2)}{x}\left(\Phi_{2}(d, x)+\Phi_{4}(d, x)\right), \\
\frac{d \Phi_{5}(d, x)}{d x}= & \frac{2(d-2)}{x}\left(\Phi_{3}(d, x)+\Phi_{5}(d, x)\right)
\end{aligned}
$$

At variance with the cases discussed in [7], [8], the system is homogeneous; indeed, quite in general the non homogeneous terms are given by the MI's of the "subtopologies" of the considered graph, obtained by shrinking to a point any of its propagator lines. When that is done for the 5-propagator "topology" of Fig.(1), one obtains the product of 4 tadpoles; but as the considered graph has two massless propagators, at least one massless tadpole is always present in the product; as in the $d$-dimensional regularization massless tadpoles vanish, the product of the 4 tadpoles is always equal to zero - and therefore the differential equations are homogeneous. 

tion

By inspection, one sees that $\Phi_{3}(d, x), \Phi_{5}(d, x)$ appear in the r.h.s. of Eq.s (2.32.7) only in the combina-

$$
\Psi_{3}(d, x)=\Phi_{3}(d, x)+\Phi_{5}(d, x)
$$

the other linearly independent combination of the two MIs, say

$$
\Psi_{5}(d, x)=\Phi_{3}(d, x)-\Phi_{5}(d, x),
$$

decouples and can be expressed in terms of the other integrals by means of the trivial 1st order differential equation

$$
\begin{aligned}
\frac{d \Psi_{5}(d, x)}{d x}= & -\left\{\frac{3(d-2)}{2(1-x)}-\frac{3(d-2)}{2(1+x)}\right\}\left(\Phi_{1}(d, x)-3 \Phi_{2}(d, x)-\Phi_{4}(d, x)\right) \\
& -\left\{\frac{5(d-2)}{x}+\frac{9(d-2)}{2(1-x)}-\frac{9(d-2)}{2(1+x)}\right\} \Psi_{3}(d, x)
\end{aligned}
$$

As $\Psi_{5}(d, x)$ does not enter in the r.h.s. of Eq.s (2.3 2.7) the 4 linear equations for $\Phi_{1}(d, x), \Phi_{2}(d, x), \Psi_{3}(d, x)$ and $\Phi_{4}(d, x)$ can be written as a fourth order equation for the first Master Integral $\Phi_{1}(x)$, which will be called simply $\Phi(d, x)$ from now on, and which is therefore equal to

$$
\Phi(d, x)=\frac{C^{-4}(d)}{(2 \pi)^{4(d-2)}} \int \frac{d^{d} k_{1} d^{d} k_{2} d^{d} k_{3} d^{d} k_{4}}{k_{1}^{2} k_{2}^{2}\left(k_{3}^{2}+1\right)\left(k_{4}^{2}+1\right)\left[\left(p-k_{1}-k_{2}-k_{3}-k_{4}\right)^{2}+x^{2}\right]}, \quad\left(p^{2}=-x^{2}\right) .
$$

One obtains for $\Phi(d, x)$ the following 4 -th order differential equation

$$
\begin{array}{r}
x^{3}\left(1-x^{2}\right) \frac{d^{4} \Phi(d, x)}{d x^{4}}+x^{2}\left\{1+5 x^{2}-3(d-4)\left(1-3 x^{2}\right)\right\} \frac{d^{3} \Phi(d, x)}{d x^{3}} \\
-x\left\{12+6 x^{2}+(d-4)\left(13+32 x^{2}\right)+(d-4)^{2}\left(1+26 x^{2}\right)\right\} \frac{d^{2} \Phi(d, x)}{d x^{2}} \\
+\left\{12-18 x^{2}+(d-4)\left(25-2 x^{2}\right)+8(d-4)^{2}\left(2+5 x^{2}\right)+3(d-4)^{3}\left(1+8 x^{2}\right)\right\} \frac{d \Phi(d, x)}{d x} \\
+4 x\left\{+12+29(d-4)+23(d-4)^{2}+6(d-4)^{3}\right\} \Phi(d, x)=0 .
\end{array}
$$


The expression of the other MI's in terms of $\Phi(d, x)$ and its first $3 x$-derivatives reads

$$
\begin{aligned}
\Phi_{2}(d, x)= & \left\{\frac{1}{2}-\frac{1}{6(d-2)}+\frac{x^{2}}{5(d-2)^{3}}-\frac{2 x^{2}}{3(d-2)^{2}}+\frac{7 x^{2}}{15(d-2)}\right\} \Phi(d, x) \\
& -\left\{\frac{x\left(1-7 x^{2}\right)}{10(d-2)^{3}}+\frac{x\left(1+16 x^{2}\right)}{12(d-2)^{2}}-\frac{x\left(11+28 x^{2}\right)}{60(d-2)}\right\} \frac{d \Phi(d, x)}{d x} \\
& -\left\{\frac{x^{2}\left(1-2 x^{2}\right)}{5(d-2)^{3}}-\frac{x^{2}\left(1-10 x^{2}\right)}{30(d-2)^{2}}\right\} \frac{d^{2} \Phi(d, x)}{d x^{2}} \\
& -\frac{x^{3}\left(1-x^{2}\right)}{20(d-2)^{3}} \frac{d^{3} \Phi(d, x)}{d x^{3}}, \\
\Psi_{3}(d, x)= & -\left\{\frac{x^{2}}{15(d-2)^{3}}-\frac{1 x^{2}}{3(d-2)^{2}}+\frac{3 x^{2}}{5(d-2)}\right\} \Phi(d, x) \\
& +\left\{\frac{x\left(1-7 x^{2}\right)}{30(d-2)^{3}}-\frac{x\left(1-8 x^{2}\right)}{12(d-2)^{2}}+\frac{x\left(1-12 x^{2}\right)}{20(d-2)}\right\} \frac{d \Phi(d, x)}{d x} \\
& +\left\{\frac{x^{2}\left(1-2 x^{2}\right)}{15(d-2)^{3}}-\frac{x^{2}\left(2-5 x^{2}\right)}{30(d-2)^{2}}\right\} \frac{d^{2} \Phi(d, x)}{d x^{2}} \\
& -\frac{x^{3}\left(1-x^{2}\right)}{60(d-2)^{3}} \frac{d^{3} \Phi(d, x)}{d x^{3}}, \\
\Phi_{4}(d, x)= & -\left\{\frac{1}{2}-\frac{1}{6(d-2)}+\frac{4 x^{2}}{5(d-2)^{3}}-\frac{3 x^{2}}{(d-2)^{2}}+\frac{43 x^{2}}{15(d-2)}\right\} \Phi(d, x) \\
& +\left\{\frac{2 x}{5(d-2)^{3}}-\frac{x\left(14-30 x^{2}\right)}{5(d-2)^{2}}-\frac{x\left(11+43 x^{2}\right)}{15(d-2)}\right\} \frac{d \Phi(d, x)}{d x} \\
& +\left\{\frac{4 x^{2}\left(1-2 x^{2}\right)}{5(d-2)^{3}}-\frac{3 x^{2}\left(1-5 x^{2}\right)}{10(d-2)^{2}}\right\} \frac{d^{2} \Phi(d, x)}{d x^{2}} \\
& +\frac{x^{3}\left(1-x^{2}\right)}{5(d-2)^{3}} \frac{d^{3} \Phi(d, x)}{d x^{3}} .
\end{aligned}
$$

\section{The $x \rightarrow 0$ behaviour of $\Phi(d, x)$}

By inspection, one finds that the most general solution of Eq.(2.12) can be expanded for $x \rightarrow 0$ in the form

$$
\Phi(d, x)=\sum_{i=1}^{4} x^{\alpha_{i}}\left(\sum_{n=0}^{\infty} A_{n}^{(i)}(d) x^{2 n}\right),
$$

where the values of the 4 exponent $\alpha_{i}$ are

$$
\begin{aligned}
& \alpha_{1}=0 \\
& \alpha_{2}=(d-2) \\
& \alpha_{3}=-(d-2) \\
& \alpha_{4}=(3 d-7) ;
\end{aligned}
$$

the $A_{0}^{(i)}(d)$ are the 4 integration constants, and all the other coefficients $A_{n}^{(i)}(d)$ for $n>0$ are determined by the differential equation Eq.(2.12) once the integration constants are fixed.

It is interesting to recall the leading exponents of the $x \rightarrow 0$ expansions of the solutions of the corresponding equations encountered in [7] and [8]. Calling $\alpha_{i}^{(2)}$ the exponents for the 2-loop graph of [7] and $\alpha_{i}^{(3)}$ those of the 3 -loop graph of [8], one finds that there are always 4 exponents. The explicit values in the 
case of $[7]$ are

$$
\begin{aligned}
\alpha_{1}^{(2)} & =0, \\
\text { - } \alpha_{2}^{(2)} & =(d-2), \\
\alpha_{3}^{(2)} & =-(d-2), \\
\alpha_{4}^{(2)} & =(d-3)
\end{aligned}
$$

and in the case of $[8]$

$$
\begin{aligned}
\alpha_{1}^{(3)} & =0 \\
-\alpha_{2}^{(3)} & =(d-2) \\
\alpha_{3}^{(3)} & =-(d-2) \\
\alpha_{4}^{(3)} & =(2 d-5)
\end{aligned}
$$

where the exponents marked by a bullet $(\bullet)$ correspond to the behaviours forced by the inhomogeneous terms. The similarity between the 3 sets of behaviours is impressive: the first 3 exponents are identical, the fourth differ in steps of $(d-2)$ for each additional loop.

In more details, 2 of the exponents of Eq.s (3.3) correspond to the 2 independent solutions of the associated homogeneous differential equation, which is of 2 nd order, while the other 2 are forced by the 2 independent behaviours for $x \rightarrow 0$ developed by the inhomogeneous terms (both products of 2 tadpoles, the first product of two tadpoles of mass $M$, the second of a tadpole of mass $M$ and a tadpole of mass $m=M x$ ). In the $x \rightarrow 0$ expansion of the most general solution, one is therefore left with 2 undetermined integration constants, corresponding to the two homogeneous solutions, while all the terms with the behaviours of the inhomogeneous terms are fully determined by the inhomogeneous equation itself. Similarly, in Eq.s(3.4) 3 exponents correspond to the 3 solutions of the 3 rd order homogeneous equation, with the 4 th exponent forced by the inhomogeneous term (product of 3 tadpoles, of masses $M, M$ and $m=M x$ ), so that there are in principle 3 undetermined integration constants, the term corresponding to the remaining behaviour being fixed by the equation. In the present case, finally, the equation is homogeneous (as already observed, there is no inhomogeneous term, as all the possible products of 4 tadpoles involve at least a vanishing zero-mass tadpole) and the 4 exponents of Eq.s (3.2) correspond to the behaviour of the 4 homogeneous solutions, so that one is left in principle with 4 undetermined integration constants.

A qualitative inspection of the integrals which one tries to evaluate by means of the differential equations (Eq. (2.11) of the present paper, Eq.(1.3) of [7] and Eq.(2.3) of [8]) shows that they are all finite (just finite, not analytic!) for $x \rightarrow 0^{+}$and $(d-2)>0$; that is sufficient to rule out from their expression as solutions of the differential equation the terms with the behaviour of the third and the fourth exponent (which is negative when $d$ is just above 2 ).

In the case of [7], that fixes completely the solution. In the case of [8], one integration constant is left undetermined; to fix it, one has to provide some independent information, such as the value of the required Feynman integral at $x=0$ (which corresponds to a simpler vacuum amplitude); that value can be provided by an explicit "conventional" calculation, say in parameter space, which is in any case much easier than a calculation for non-zero values of the variable $x$. (A closer analysis carried out in [8] shows however that the regularity of the integral at $x=1$ is sufficient to fully determine the solution, so that the actual knowledge of the $x=0$ value can be used as an independent check).

In the current case, as the equation for $\Phi(d, x)$ is homogeneous, the only information is that $A_{0}^{(3)}(d)$ and $A_{0}^{(4)}(d)$ are both equal to zero due to the finiteness for $x \rightarrow 0^{+}$; by substituting the ansatz Eq.(13.1) in Eq.(2.12) and dropping $A_{0}^{(3)}(d), A_{0}^{(4)}(d)$, one finds for $\Phi(d, x)$ Eq. (2.11) the $x \rightarrow 0$ expansion

$$
\begin{aligned}
\Phi(d, x) & =A_{0}^{(1)}(d)\left(1-\frac{2(2 d-5)(3 d-8)}{3 d(d-4)} x^{2}+O\left(x^{4}\right)\right) \\
& +A_{0}^{(2)}(d) x^{d-2}\left(1+\frac{(d-3)(d-4)(3 d-8)}{2 d(2 d-7)} x^{2}+O\left(x^{4}\right)\right) .
\end{aligned}
$$


The expansion depends on the two as yet unspecified integrations constants $A_{0}^{(1)}(d), A_{0}^{(2)}(d)$ - which are to be provided by an independent, explicit calculation. That is done in Section [7 see Eq.(17.14), by direct integration in the parametric space.

Let us note here, for completeness, that in the present case the knowledge of the regularity of the solution at $x=1$ does not provide any additional information.

\section{The expansion in $(d-4)$ and the homogeneous equation at $d=4$.}

The Laurent's expansion in $(d-4)$ of $\Phi(d, x)$ Eq.(2.11) is

$$
\Phi(d, x)=\sum_{n=-4}^{\infty}(d-4)^{n} \Phi^{(n)}(x),
$$

as it is known on general grounds that it develops at most a fourth order pole in $(d-4)$. By substituting in Eq. (2.12) one obtains a system of inhomogeneous, chained equations for the coefficients $\Phi^{(n)}(x)$ of the expansion in $(d-4)$; the generic equation reads

$$
\left[x^{3}\left(1-x^{2}\right) \frac{d^{4}}{d x^{4}}+x^{2}\left(1+5 x^{2}\right) \frac{d^{3}}{d x^{3}}-6 x\left(2+x^{2}\right) \frac{d^{2}}{d x^{2}}+6\left(2-3 x^{2}\right) \frac{d}{d x}+48 x\right] \Phi^{(n)}(x)=K^{(n)}(x),
$$

with

$$
\begin{aligned}
K^{(n)}(x)= & \left\{24 x+\left(3+24 x^{2}\right) \frac{d}{d x}\right\} \Phi^{(n-3)}(x) \\
& +\left\{92 x+\left(16+40 x^{2}\right) \frac{d}{d x}-\left(x+26 x^{3}\right) \frac{d^{2}}{d x^{2}}\right\} \Phi^{(n-2)}(x) \\
& +\left\{116 x+\left(25-2 x^{2}\right) \frac{d}{d x}-\left(13 x+32 x^{3}\right) \frac{d^{2}}{d x^{2}}-\left(3 x^{2}-9 x^{4}\right) \frac{d^{3}}{d x^{3}}\right\} \Phi^{(n-1)}(x),
\end{aligned}
$$

which shows that the equation at a given order $n$ for $\Phi^{(n)}(x)$ involves in the inhomogeneous term the coefficients $\Phi^{(k)}(x)$ (and their derivatives) with $k<n$ - hence the "chained equations" expression used above (obviously $\Phi^{(k)}(x)=0$ when $k<-4$ ). Such a structure calls for an algorithm of solution bottom-up, i.e. starting from the lowest value of $n$ (which is $n=-4$ ) and proceeding recursively to the next $n+1$ value up to the required order.

The Eq.s (4.2) have all the same associated homogeneous equation, independent of $n$,

$$
\left[x^{3}\left(1-x^{2}\right) \frac{d^{4}}{d x^{4}}+x^{2}\left(1+5 x^{2}\right) \frac{d^{3}}{d x^{3}}-6 x\left(2+x^{2}\right) \frac{d^{2}}{d x^{2}}+6\left(2-3 x^{2}\right) \frac{d}{d x}+48 x\right] \phi(x)=0 ;
$$

once the solutions of Eq. (4.4) are known, all the Eq.s (4.2) can be solved by the method of the variation of the constants of Euler.

To our (pleasant) surprise, the solutions of Eq.(4.4) are almost elementary. By trial and error, a first solution is found to be

$$
\phi_{1}(x)=x^{2} .
$$

We then substitute $\phi(x)=\phi_{1}(x) \xi(x)$ in Eq.(4.4), obtaining the following 3rd order equation for the derivative of $\xi(x)$

$$
\left[x^{3}\left(1-x^{2}\right) \frac{d^{3}}{d x^{3}}+3 x^{2}\left(3-x^{2}\right) \frac{d^{2}}{d x^{2}}+6 x\left(1+2 x^{2}\right) \frac{d}{d x}-6\left(5+2 x^{2}\right)\right] \xi^{\prime}(x)=0,
$$

and find that it admits the solution

$$
\xi_{2}^{\prime}(x)=\frac{1}{x^{3}}\left(1-x^{2}+x^{4}\right)
$$


Substituting $\xi^{\prime}(x)=\xi_{2}^{\prime}(x) \chi(x)$ in Eq.(4.6) we obtain the following 2nd order equation for $\chi^{\prime}(x)$

$$
\left[x^{2}\left(1-x^{2}\right)\left(1-x^{2}+x^{4}\right) \frac{d^{2}}{d x^{2}}+6 x^{5}\left(2-x^{2}\right) \frac{d}{d x}-6\left(2-2 x^{4}+x^{6}\right)\right] \chi^{\prime}(x)=0,
$$

which admits as solution

$$
\chi_{3}^{\prime}(x)=\frac{1}{x^{3}}\left(1-x^{2}\right)^{4} \frac{5-2 x^{2}+5 x^{4}}{\left(1-x^{2}+x^{4}\right)^{2}} .
$$

Finally, substituting $\chi^{\prime}(x)=\chi_{3}^{\prime}(x) \tau(x)$ in Eq.(4.8), we obtain the equation

$$
\begin{aligned}
& {\left[x\left(1-x^{2}\right)^{5}\left(1-x^{2}+x^{4}\right)\left(5-2 x^{2}+5 x^{4}\right) \frac{d}{d x}\right.} \\
& \left.-2\left(1-x^{2}\right)^{4}\left(15-12 x^{2}+11 x^{4}+30 x^{6}-24 x^{8}+20 x^{10}\right)\right] \tau^{\prime}(x)=0,
\end{aligned}
$$

which has the solution

$$
\tau_{4}^{\prime}(x)=\frac{x^{6}}{\left(1-x^{2}\right)^{5}} \frac{1-x^{2}+x^{4}}{5-2 x^{2}+5 x^{4}} .
$$

By repeated quadratures in $x$ and multiplications by the previous solutions we obtain the explicit analytic expressions of the 4 solutions of Eq.(4.4); the nasty denominators $\left(1-x^{2}+x^{4}\right)$ and $\left(5-2 x^{2}+5 x^{4}\right)$ disappear in the final results, while the repeated integrations of the terms with denominators $x,(1+x)$ and $(1-x)$ generate, almost by definition, Harmonic PolyLogarithms [10] of argument $x$ and weight increasing up to 3. Explicitly, one finds

$$
\begin{aligned}
\phi_{2}(x)= & -\frac{1}{2}\left(1-x^{4}\right)-H(0, x) x^{2} \\
\phi_{3}(x)= & \frac{\left(5+18 x^{2}+14 x^{6}+5 x^{8}\right)}{8 x^{2}}+\frac{1}{2}\left(12+x^{2}-12 x^{4}\right) H(0 ; x)+12 x^{2} H(0,0 ; x), \\
\phi_{4}(x)= & \frac{\left(1+x^{2}\right)\left(15+182 x^{2}+15 x^{4}\right)}{65536 x}+\frac{3\left(1-x^{2}\right)^{2}\left(5+x^{2}\right)\left(1+5 x^{2}\right)}{131072 x^{2}}[H(-1 ; x)+H(1 ; x)] \\
& -\frac{9\left(1-x^{4}\right)}{8192}[H(0,-1 ; x)+H(0,1 ; x)]-\frac{9 x^{2}}{4096}[H(0,0,-1 ; x)+H(0,0,1 ; x)] .
\end{aligned}
$$

The corresponding Wronskian has the remarkably simple expression

$$
W(x)=\left|\begin{array}{cccc}
\phi_{1}(x) & \phi_{2}(x) & \phi_{3}(x) & \phi_{4}(x) \\
\phi_{1}^{\prime}(x) & \phi_{2}^{\prime}(x) & \phi_{3}^{\prime}(x) & \phi_{4}^{\prime}(x) \\
\phi_{1}^{\prime \prime}(x) & \phi_{2}^{\prime \prime}(x) & \phi_{3}^{\prime \prime}(x) & \phi_{4}^{\prime \prime}(x) \\
\phi_{1}^{\prime \prime \prime}(x) & \phi_{2}^{\prime \prime \prime}(x) & \phi_{3}^{\prime \prime \prime}(x) & \phi_{4}^{\prime \prime \prime}(x)
\end{array}\right|=\frac{\left(1-x^{2}\right)^{3}}{x},
$$

in agreement (of course) with the coefficients of the $4^{\text {th }}$ and $3^{r d} x$-derivative of $\phi(x)$ in Eq. (4.4).

\section{The Solution of the differential equations for the coefficients of the expansion in $(d-4)$.}

With the results established in the previous Section one can use Euler's method of the variation of the constants for solving Eq.s(4.2) recursively in $n$, starting from $n=-4$. We write Euler's formula as

$$
\Phi^{(n)}(x)=\sum_{i=1}^{4} \phi_{i}(x)\left[\Phi_{i}^{(n)}+\int_{0}^{x} \frac{d x^{\prime}}{W\left(x^{\prime}\right)} M_{i}\left(x^{\prime}\right) K^{(n)}\left(x^{\prime}\right)\right]
$$


where the $\phi_{i}(x)$ are the solutions of the homogeneous equation given in Eq.s (4.54.14), the $\Phi_{i}^{(n)}$ are the as yet undetermined integration constants, the Wronskian $W(x)$ can be read from Eq. (4.15), the $M_{i}(x)$ are the minors of the $\phi_{i}^{\prime \prime \prime}(x)$ in the determinant Eq. (4.15), and the $K^{(n)}(x)$ are the inhomogeneous terms of Eq.(4.3). The constants $\Phi_{i}^{(n)}$ are then fixed by comparing the expansion in $x$ for $x \rightarrow 0$ of Eq. (5.1) with the expansion in $(d-4)$ for $d \rightarrow 4$ of Eq. (3.5. Explicitly, we find for instance

$$
\begin{aligned}
\Phi^{(-4)}(x)= & -\frac{1}{64} x^{2} \\
\Phi^{(-3)}(x)= & -\frac{1}{384}+\frac{9}{256} x^{2}-\frac{1}{192} x^{4}-\frac{1}{48} x^{2} H(0 ; x) \\
\Phi^{(-2)}(x)= & \frac{41}{4608}-\frac{143}{3072} x^{2}+\frac{37}{2304} x^{4}-\frac{5}{9216} x^{6}+\left(\frac{3}{64}-\frac{1}{96} x^{2}\right) x^{2} H(0 ; x)-\frac{1}{48} x^{2} H(0,0 ; x) \\
\Phi^{(-1)}(x)= & -\frac{805}{55296}+\frac{205}{4096} x^{2}-\frac{743}{27648} x^{4}+\frac{599}{221184} x^{6}-\left(\frac{43}{768}-\frac{11}{384} x^{2}+\frac{5}{2304} x^{4}\right) x^{2} H(0 ; x) \\
& +\left(\frac{3}{64}-\frac{1}{96} x^{2}\right) x^{2} H(0,0 ; x)-\frac{1}{48} x^{2} H(0,0,0 ; x) \\
\Phi^{(0)}(x)= & \frac{3173}{663552}-\frac{1}{3840} \pi^{4} x^{2}-\frac{2443}{49152} x^{2}-\frac{1}{96} x^{4} \zeta(3)+\frac{15649}{331776} x^{4}-\frac{34061}{5308416} x^{6}+\frac{1}{96} \zeta(3) \\
& -\left(\frac{5}{1536}-\frac{1}{48} \zeta(3) x^{2}+\frac{1}{768} x^{2}+\frac{121}{2304} x^{4}-\frac{599}{55296} x^{6}\right) H(0 ; x)+\left(\frac{1}{96}+\frac{5}{1536} \frac{1}{x^{2}}\right. \\
& \left.-\frac{7}{256} x^{2}+\frac{1}{96} x^{4}+\frac{5}{1536} x^{6}\right)[H(1,0 ; x)-H(-1,0 ; x)]-\left(\frac{43}{768} x^{2}-\frac{11}{384} x^{4}\right. \\
& \left.+\frac{5}{2304} x^{6}\right) H(0,0 ; x)+\frac{\left(1-x^{4}\right)}{32}[H(0,1,0 ; x)-H(0,-1,0 ; x)]+\frac{1}{16} x^{2}[H(0,0,1,0 ; x) \\
& -H(0,0,-1,0 ; x)]+\left(\frac{3}{64}-\frac{1}{96} x^{2}\right) x^{2} H(0,0,0 ; x)-\frac{1}{48} x^{2} H(0,0,0,0 ; x) .
\end{aligned}
$$

The full results become quickly too lengthy to be reported explicitly here, so we give only the values of the integration constants up to order 3 included. We find $\Phi_{4}^{(k)}=0$, for (all) $-4 \leq k \leq 3$; the other constants 
are

$$
\begin{aligned}
\Phi_{1}^{(1)}= & \frac{24978775}{603979776}+\frac{32419}{786432} \zeta(3)+\frac{4181}{5242880} \pi^{4}-\frac{7}{64} \zeta(5) ; \\
\Phi_{2}^{(1)}= & -\frac{19014089}{905969664}+\frac{34481}{393216} \zeta(3)-\frac{8507}{7864320} \pi^{4} ; \\
\Phi_{3}^{(1)}= & \frac{264575}{452984832}-\frac{871}{196608} \zeta(3)-\frac{19}{3932160} \pi^{4} ; \\
\Phi_{1}^{(2)}= & \frac{1791217627}{7247757312}-\frac{476887}{3145728} \zeta(3)+\frac{37601}{62914560} \pi^{4}+\frac{87801}{262144} \zeta(5)+\frac{1}{32} \zeta^{2}(3)-\frac{13}{32256} \pi^{6} ; \\
\Phi_{2}^{(2)}= & \frac{674733523}{10871635968}-\frac{691111}{4718592} \zeta(3)+\frac{79069}{18874368} \pi^{4}-\frac{43165}{131072} \zeta(5) ; \\
\Phi_{3}^{(2)}= & -\frac{191322157}{5435817984}+\frac{27041}{2359296} \zeta(3)-\frac{1787}{9437184} \pi^{4}-\frac{133}{65536} \zeta(5) ; \\
\Phi_{1}^{(3)}= & -\frac{40220928899}{21743271936}+\frac{6041939}{9437184} \zeta(3)-\frac{775961}{150994944} \pi^{4}+\frac{3855}{1048576} \zeta(5)-\frac{12543}{131072} \zeta^{2}(3) \\
& +\frac{54353}{44040192} \pi^{6}+\frac{1}{384} \zeta(3) \pi^{4}-\frac{605}{512} \zeta(7) ; \\
\Phi_{2}^{(3)}= & \frac{336259373}{16307453952}+\frac{1366211}{14155776} \zeta(3)-\frac{7536877}{1132462080} \pi^{4}+\frac{1939871}{1572864} \zeta(5)+\frac{15281}{196608} \zeta^{2}(3) \\
& -\frac{69631}{66060288} \pi^{6} ; \\
\Phi_{3}^{(3)}= & \frac{3741658007}{16307453952}-\frac{247061}{7077888} \zeta(3)+\frac{275627}{566231040} \pi^{4}-\frac{41641}{786432} \zeta(5)+\frac{19}{32768} \zeta^{2}(3) \\
& \left.-\frac{2430144}{6}\right) \\
= &
\end{aligned}
$$

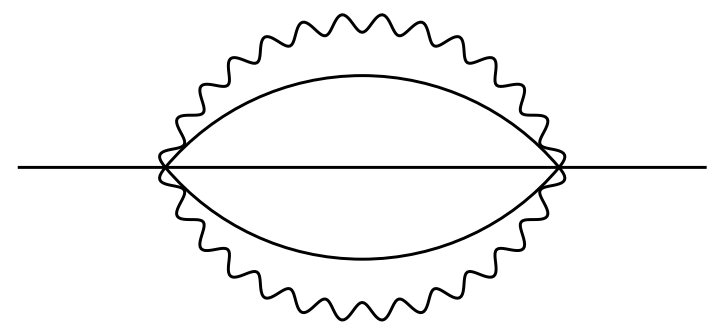

Figure 2: The on-shell 4-loop sunrise graph.

As a byproduct, using the results of [12], we obtain the values at $x=1$, i.e. the on mass-shell values 
at $-p^{2}=m^{2}=M^{2}$ of Eq.(2.1), depicted in Fig.2,

$$
\begin{aligned}
\Phi(d, x=1)= & -\frac{1}{64(d-4)^{4}}+\frac{7}{256(d-4)^{3}}-\frac{17}{768(d-4)^{2}}+\frac{835}{73728(d-4)}-\frac{7379}{1769472}-\frac{1}{1920} \pi^{4} \\
+ & (d-4)\left\{-\frac{6766055}{42467328}+\frac{53}{1152} \pi^{2}+\frac{7}{7680} \pi^{4}-\frac{7}{256} \pi^{2} \zeta(3)+\frac{29}{256} \zeta(5)\right\} \\
+(d-4)^{2}\{ & \frac{1449210865}{1019215872}-\frac{1913}{4608} \pi^{2}+\frac{53}{128} \pi^{2} \ln 2-\frac{1855}{2304} \zeta(3)-\frac{17}{23040} \pi^{4}+\frac{49}{1024} \pi^{2} \zeta(3) \\
& -\frac{203}{1024} \zeta(5)-\frac{63}{256} \pi^{2} \zeta(3) \ln 2-\frac{9}{32} a_{4} \pi^{2}-\frac{3}{256} \pi^{2} \ln ^{4} 2+\frac{3}{256} \pi^{4} \ln ^{2} 2 \\
& \left.+\frac{245}{1024} \zeta^{2}(3)+\frac{661}{322560} \pi^{6}\right\} \\
+(d-4)^{3}\{ & -\frac{182188906799}{24461180928}+\frac{734695}{331776} \pi^{2}-\frac{1913}{512} \pi^{2} \ln 2+\frac{66955}{9216} \zeta(3)+\frac{583}{384} \pi^{2} \ln 22 \\
& -\frac{8707}{147456} \pi^{4}+\frac{265}{768} \ln 2+\frac{265}{32} a_{4}-\frac{119}{3072} \pi^{2} \zeta(3)+\frac{493}{3072} \zeta(5)+\frac{441}{1024} \pi^{2} \zeta(3) \ln 2 \\
& +\frac{21}{1024} \pi^{2} \ln { }^{4} 2-\frac{21}{1024} \pi^{4} \ln ^{2} 2-\frac{661}{184320} \pi^{6}+\frac{63}{128} a_{4} \pi^{2}-\frac{1715}{4096} \zeta^{2}(3) \\
& -\frac{627}{512} \pi^{2} \zeta(3) \ln 2-\frac{123}{1280} \pi^{2} \ln ^{5} 2+\frac{9917}{4096} \pi^{2} \zeta(5)+\frac{107}{1536} \pi^{4} \ln ^{3} 2 \\
& -\frac{109}{12288} \pi^{4} \zeta(3)+\frac{2205}{1024} \zeta^{2}(3) \ln 2+\frac{45}{128} \zeta(3) \ln ^{4} 2-\frac{15}{2048} \pi^{6} \ln ^{2} \\
& -\frac{81}{32} a_{4} \pi^{2} \ln 2+\frac{45}{16} a_{4} \zeta(3)-\frac{81}{32} a_{5} \pi^{2}-\frac{45}{16} a_{5} \ln ^{2} 2+\frac{1395}{1024} \zeta(5) \ln ^{2} 2 \\
& \left.-\frac{45}{4} a_{6} \ln 2+\frac{45}{16} b_{6} \ln 2-\frac{135}{8} a_{7}+\frac{45}{8} b_{7}-\frac{45}{32} d_{7}+\frac{15}{1792} \ln ^{7} 2+\frac{29335}{2048} \zeta(7)\right\} \\
+\mathcal{O}\left((d-4)^{4}\right) &
\end{aligned}
$$

At variance with [12], we have expressed the results in terms of the constants listed in Table 1; the first column is the name of the constant, the second column its value as Harmonic PolyLogarithm of suitable argument, the third as Nielsen PolyLogarithm (when available), the last the numerical value.

\begin{tabular}{|c|c|c|l|}
\hline constant & HPL & NPl & numerical value \\
\hline \hline$\zeta(3)$ & $H(0,0,1 ; 1)$ & $S_{2,1}(1)$ & 1.2020569031595942854 \\
$a_{4}$ & $H(0,0,0,1 ; 1 / 2)$ & $S_{3,1}(1 / 2)$ & 0.51747906167389938633 \\
$\zeta(5)$ & $H(0,0,0,0,1 ; 1)$ & $S_{4,1}(1)$ & 1.0369277551433699263 \\
$a_{5}$ & $H(0,0,0,0,1 ; 1 / 2)$ & $S_{4,1}(1 / 2)$ & 0.50840057924226870746 \\
$a_{6}$ & $H(0,0,0,0,0,1 ; 1 / 2)$ & $S_{5,1}(1 / 2)$ & 0.50409539780398855069 \\
$b_{6}$ & $H(0,0,0,0,1,1 ; 1 / 2)$ & $S_{4,2}(1 / 2)$ & 0.0087230030575968884272 \\
$\zeta(7)$ & $H(0,0,0,0,0,0,1 ; 1)$ & $S_{6,1}(1)$ & 1.0083492773819228268 \\
$a_{7}$ & $H(0,0,0,0,0,0,1 ; 1 / 2)$ & $S_{6,1}(1 / 2)$ & 0.50201456332470849457 \\
$b_{7}$ & $H(0,0,0,0,0,1,1 ; 1 / 2)$ & $S_{5,2}(1 / 2)$ & 0.0041965726953603256975 \\
$d_{7}$ & $H(0,0,0,0,1,-1,-1 ; 1)$ & -- & 0.0022500546439578516764 \\
\hline
\end{tabular}

Table 1: Constants up to weight 7 appearing in Eq.(5.16)

Once the explicit analytic expressions of the coefficients of the Laurent-expansion of $\Phi(d, x)$ in $(d-4)$ are known, one can use Eq.s 2.132.15) for obtaining the coefficients of $\Phi_{2}(d, x), \Psi_{3}(d, x)$ and $\Phi_{4}(d, x)$. The coefficients of the expansion of $\Psi_{5}(d, x)$ are then recovered by integrating in $x$ Eq.(2.10); the quadrature is trivial to carry out in terms of HPLs, and the integration constants are fixed by the condition $\Psi_{5}(d, 0)=0$, which follows at once from Eq.s(2.9), (2.2), (2.1). 


\section{The Independent numerical calculation.}

In this Section we will calculate $\Phi(d, x=1)$ with high numerical precision by suitably using the difference equation method described in $[5,6]$ and references therein. At variance with [6], we will apply the method directly to the Master Integral with massless lines. This will imply some additional work for establishing the initial conditions of the difference equations.

We define

$$
I_{5}(d, n)=\pi^{-2 d} \int \frac{d^{d} k_{1} d^{d} k_{2} d^{d} k_{3} d^{d} k_{4}}{\left(k_{1}^{2}+1\right)^{n}\left(\left(k_{2}-k_{1}\right)^{2}+1\right)\left(\left(k_{3}-k_{2}\right)^{2}+1\right)\left(k_{4}-k_{3}\right)^{2}\left(p-k_{4}\right)^{2}}, \quad p^{2}=-1,
$$

so that $I_{5}(d, 1)$ is equal to $\Phi(d, x)$ of Eq.(2.11) at $x=1$ up to a known multiplicative factor

$$
I_{5}(d, 1)=[4 \Gamma(1+\epsilon)]^{4} \Phi(d=4-2 \epsilon, x=1) .
$$

By combining identities obtained by integration by parts one finds that $I_{5}(d, n)$ satisfies the third-order difference equation

$$
\begin{array}{r}
32(n-1)(n-2)(n-3)(n-3 d+5) I_{5}(d, n) \\
-4(n-2)(n-3)\left[15 n^{2}+(39-50 d) n+27 d^{2}+5 d-54\right] I_{5}(d, n-1) \\
+2(n-3)\left[12 n^{3}-(38 d+24) n^{2}+\left(23 d^{2}+133 d-84\right) n+9 d^{3}-141 d^{2}+134 d-24\right] I_{5}(d, n-2) \\
+(n-d-1)(n-2 d+1)(2 n-3 d)(2 n-5 d+4) I_{5}(d, n-3)=0
\end{array}
$$

We will solve this difference equation by using the Laplace ansatz

$$
I_{5}(d, n)=\int_{0}^{1} t^{n-1} v_{5}(d, t) d t,
$$

giving for $v_{5}(d, t)$ the fourth-order differential equation

$$
\begin{aligned}
& 4 t^{4}(8 t+1)(t-1)^{2} \frac{d^{4}}{d t^{4}} v_{5}(d, t) \\
& +4 t^{3}(t-1)\left[24(d+1) t^{2}+(18-26 d) t-7 d+12\right] \frac{d^{3}}{d t^{3}} v_{5}(d, t) \\
& t^{2}\left[\left(576(d-1) t^{3}+\left(-108 d^{2}-420 d+648\right) t^{2}+\left(46 d^{2}+38 d-144\right) t+71 d^{2}-284 d+288\right] \frac{d^{2}}{d t^{2}} v_{5}(d, t)\right. \\
& +t\left[(576 d-960) t^{3}+\left(-216 d^{2}+360 d\right) t^{2}\right. \\
& \left.\left.+\left(-18 d^{3}+190 d^{2}-496 d+384\right) t+77 d^{3}-533 d^{2}+1236 d-960\right)\right] \frac{d}{d t} v_{5}(d, t) \\
& +(d-3)(2 d-5)(3 d-8)(5 d-12) v_{5}(d, t)=0 .
\end{aligned}
$$

We will look for the solution of Eq.(6.5) in the form of a power series expansions; inserted in Eq.(6.4) and integrated term by term it will provide very accurate values of $I_{5}(d, n)$. As the convergence is faster for larger $n$, we will consider "large enough" values of the index $n$ (see below); the repeated use "top-down" of Eq.6.3) (i.e. using it for expressing $I_{5}(d, n-3)$ in terms of the $I_{5}(k)$ with $\left.k=n, n-1, n-2\right)$ will give the values corresponding to smaller indices, till $I_{5}(d, 1)$ is eventually obtained. To go on with this program, initial conditions for $v_{5}(d, t)$ are needed. 
From the definition Eq. (6.1), and introducing spherical coordinates in $d$-dimension for the loop momentum $k_{1}, d^{d} k_{1}=k_{1}^{d-1} d k_{1} d \Omega\left(d, \hat{k}_{1}\right)$ one has

$$
\begin{aligned}
I_{5}(d, n) & =\frac{1}{\Gamma\left(\frac{d}{2}\right)} \int_{0}^{\infty} \frac{\left(k_{1}^{2}\right)^{d / 2-1} d k_{1}^{2}}{\left(k_{1}^{2}+1\right)^{n}} f_{5}\left(d, k_{1}^{2}\right), \\
f_{5}\left(d, k_{1}^{2}\right) & =\int \frac{d \Omega\left(d, \hat{k}_{1}\right)}{\Omega(d)} I_{4}\left(d, 1,\left(p-k_{1}\right)^{2}\right),
\end{aligned}
$$

where $\Omega(d)$ is the $d$-dimensional solid angle, and $I_{4}\left(d, 1,\left(p-k_{1}\right)^{2}\right)$ is the 3-loop (off mass-shell) sunrise integral

$$
I_{4}\left(d, n,\left(p-k_{1}\right)^{2}\right)=\pi^{-3 d / 2} \int \frac{d^{d} k_{2} d^{d} k_{3} d^{d} k_{4}}{\left(\left(k_{2}-k_{1}\right)^{2}+1\right)^{n}\left(\left(k_{3}-k_{2}\right)^{2}+1\right)\left(k_{4}-k_{3}\right)^{2}\left(p-k_{4}\right)^{2}} .
$$

By the change of variable $1 /\left(k_{1}^{2}+1\right)=t, \quad k_{1}^{2}=(1-t) / t$, one finds

$$
I_{5}(d, n)=\frac{1}{\Gamma\left(\frac{d}{2}\right)} \int_{0}^{1} t^{n-1}(1-t)^{\frac{d}{2}-1} t^{-\frac{d}{2}} f_{5}\left(d, \frac{1-t}{t}\right) d t,
$$

from which one gets the relation between $v_{5}(d, t)$ and $f_{5}(d,(1-t) / t)$

$$
v_{5}(d, t)=\frac{1}{\Gamma\left(\frac{d}{2}\right)}(1-t)^{\frac{d}{2}-1} t^{-\frac{d}{2}} f_{5}\left(d, \frac{1-t}{t}\right) d t .
$$

From that relation we see that we can derive boundary conditions for $v_{5}(d, t)$ in the $t \rightarrow 1$ limit from the expansion of $f_{5}\left(d, k_{1}^{2}\right)$ in the $k_{1} \rightarrow 0$ limit, which is easy to obtain. Indeed, only the first denominator of Eq. (6.8) depends on $k_{1}$; expanding for small $k_{1}$ and performing the angular integration one gets

$$
\begin{aligned}
\int \frac{d \Omega\left(d, \hat{k}_{1}\right)}{\Omega(d)} \frac{1}{\left(k_{2}-k_{1}\right)^{2}+1} & =\int \frac{d \Omega\left(d, \hat{k}_{1}\right)}{\Omega(d)}\left(\frac{1}{k_{2}^{2}+1}-\frac{k_{1}^{2}-2 k_{1} \cdot k_{2}}{\left(k_{2}^{2}+1\right)^{2}}+\frac{\left(k_{1}^{2}-2 k_{1} \cdot k_{2}\right)^{2}}{\left(k_{2}^{2}+1\right)^{3}}+\ldots\right) \\
& =\frac{1}{k_{2}^{2}+1}+k_{1}^{2}\left(-\frac{1}{\left(k_{2}^{2}+1\right)^{2}}+\frac{4}{d} \frac{k_{2}^{2}}{\left(k_{2}^{2}+1\right)^{3}}\right)+\ldots
\end{aligned}
$$

The above result gives the expansion of $f_{5}\left(d, k_{1}^{2}\right)$ at $k_{1}^{2}=0$ :

$$
\begin{aligned}
f_{5}\left(d, k_{1}^{2}\right) & =f_{5}^{(0)}(d)+f_{5}^{(1)}(d) k_{1}^{2}+O\left(k_{1}^{4}\right) \\
f_{5}^{(0)}(d) & =I_{4}\left(d, 1, p^{2}\right) \\
f_{5}^{(1)}(d) & =-I_{4}\left(d, 2, p^{2}\right)+\frac{4}{d}\left[I_{4}\left(d, 2, p^{2}\right)-I_{4}\left(d, 3, p^{2}\right)\right] .
\end{aligned}
$$

Note that $f_{5}\left(d, k_{1}^{2}\right)$ is regular in the origin.

By inspecting the differential equation (6.5) one finds that the behaviour at $t=1$ of the 4 independent solution is $\sim(1-t)^{\alpha_{i}}$, with $\alpha_{1}=d / 2-1, \alpha_{2}=d / 2, \alpha_{3}=0$, and $\alpha_{4}=1$; for comparison with Eq. (6.10) the behaviours $\alpha_{3}=0$, and $\alpha_{4}=1$ are ruled out and the expansion reads

$$
v_{5}(d, t)=(1-t)^{\frac{d}{2}-1}\left(v_{5}^{(0)}(d)+v_{5}^{(1)}(d)(1-t)+O(1-t)^{2}\right) ;
$$

by comparison with Eq.(6.12) $\left(t=1\right.$ corresponds to $\left.k_{1}^{2}=0\right)$, one obtains

$$
\begin{aligned}
& v_{5}^{(0)}(d)=\frac{1}{\Gamma\left(\frac{d}{2}\right)} f_{5}^{(0)}(d), \\
& v_{5}^{(1)}(d)=\frac{1}{\Gamma\left(\frac{d}{2}\right)}\left[\frac{d}{2} f_{5}^{(0)}(d)+f_{5}^{(1)}(d)\right] .
\end{aligned}
$$


The values $I_{4}(d, n)$ of $I_{4}\left(d, n, p^{2}\right)$ at $p^{2}=-1$ are therefore required

$$
I_{4}(d, n) \equiv I_{4}\left(d, n, p^{2}\right)=\pi^{-3 d / 2} \int \frac{d^{d} k_{2} d^{d} k_{3} d^{d} k_{4}}{\left(k_{2}^{2}+1\right)^{n}\left(\left(k_{3}-k_{2}\right)^{2}+1\right)\left(k_{4}-k_{3}\right)^{2}\left(p-k_{4}\right)^{2}}, \quad p^{2}=-1 .
$$

The problem of evaluating the $I_{4}(d, n)$ is similar to the original problem of evaluating the $I_{5}(d, n)$, but in fact it is much simpler, as the $I_{4}(d, n)$ involve one less loop and one less propagator. As above, by using integration-by-parts identities one finds that $I_{4}(d, n)$ satisfies the third-order difference equation

$$
\begin{aligned}
& 6(n-1)(n-2)(n-3) I_{4}(d, n) \\
& -(n-2)(n-3)(10 n-7 d-10) I_{4}(d, n-1) \\
& +(n-3)\left(2 n^{2}+(2 d-18) n-7 d^{2}+29 d-8\right) I_{4}(d, n-2) \\
& +(n-d-1)(n-2 d+1)(2 n-3 d) I_{4}(d, n-3)=0 .
\end{aligned}
$$

We solve the difference equation by using again the Laplace ansatz

$$
I_{4}(d, n)=\int_{0}^{1} t^{n-1} v_{4}(d, t) d t,
$$

where $v_{4}(d, t)$ satisfies the differential equation

$$
\begin{aligned}
& 2 t^{3}(3 t+1)(t-1)^{2} \frac{d^{3}}{d t^{3}} v_{4}(d, t) \\
& +t^{2}(t-1)\left(36 t^{2}+(6-7 d) t-9 d+18\right) \frac{d^{2}}{d t^{2}} v_{4}(d, t) \\
& +t\left(36 t^{3}-14 d t^{2}+\left(-7 d^{2}+33 d-36\right) t+13 d^{2}-61 d+72\right) \frac{d}{d t} v_{4}(d, t) \\
& +(d-3)(2 d-5)(3 d-8) v_{4}(d, t)=0 .
\end{aligned}
$$

Following the procedure used above, we write

$$
\begin{aligned}
I_{4}(d, n) & =\frac{1}{\Gamma\left(\frac{d}{2}\right)} \int_{0}^{\infty} \frac{\left(k_{2}^{2}\right)^{d / 2-1} d k_{2}^{2}}{\left(k_{2}^{2}+1\right)^{n}} f_{4}\left(k_{2}^{2}\right), \\
f_{4}\left(k_{2}^{2}\right) & =\int \frac{d \Omega\left(d, \hat{k}_{2}\right)}{\Omega(d)} I_{3}\left(d,\left(p-k_{2}\right)^{2}\right), \\
I_{3}\left(d,\left(p-k_{2}\right)^{2}\right) & =\pi^{-d} \int \frac{d^{d} k_{3} d^{d} k_{4}}{\left(\left(k_{3}-k_{2}\right)^{2}+1\right)\left(k_{4}-k_{3}\right)^{2}\left(p-k_{4}\right)^{2}}, \\
v_{4}(d, t) & =\frac{1}{\Gamma\left(\frac{d}{2}\right)}(1-t)^{\frac{d}{2}-1} t^{-\frac{d}{2}} f_{4}\left(d, \frac{1-t}{t}\right) .
\end{aligned}
$$

At variance with the previous case, the function $f_{4}\left(d, k_{2}^{2}\right)$ is not regular for $k_{2} \rightarrow 0$, as at $k_{2}=0$ the value of the external momentum squared $\left(p-k_{2}\right)^{2}$ becomes the threshold of the 2-loop sunrise graph associated to $I_{3}\left(d, p^{2}\right)$. But it is not difficult to evaluate analytically $I_{3}\left(d, q^{2}\right)$ for generic off-shell $q^{2}$ by using Feynman parameters:

$$
I_{3}\left(d, q^{2}\right)=\frac{2 \Gamma(5-d) \Gamma\left(3-\frac{d}{2}\right) \Gamma^{2}\left(\frac{d}{2}-1\right)}{(d-4)^{2}(3-d) \Gamma\left(\frac{d}{2}\right)}{ }_{2} F_{1}\left(3-d, 2-\frac{d}{2} ; \frac{d}{2} ;-q^{2}\right),
$$

where ${ }_{2} F_{1}$ is the Gauss hypergeometric function. The expansion of $I_{3}\left(d, q^{2}\right)$ in $q^{2}=-1$ consists of the sum of two series,

$$
I_{3}\left(d, q^{2}\right)=a_{0}(d)\left[1+O\left(q^{2}+1\right)\right]+b_{0}(d)\left(q^{2}+1\right)^{2 d-5}\left[1+O\left(q^{2}+1\right)\right]
$$




$$
\begin{aligned}
a_{0}(d)=I_{3}(d,-1) & =\frac{2 \Gamma(5-d) \Gamma\left(3-\frac{d}{2}\right) \Gamma^{2}\left(\frac{d}{2}-1\right) \Gamma(2 d-5)}{(4-d)^{2}(3-d) \Gamma\left(\frac{3}{2} d-3\right) \Gamma(d-2)}, \\
b_{0}(d) & =\Gamma^{2}\left(\frac{d}{2}-1\right) \Gamma(5-2 d) .
\end{aligned}
$$

Inserting Eq.(6.23) into Eq.(6.20), setting $q=p-k_{2}$ and performing the angular integration over $\hat{k}_{2}$ by means of the formula (see Eq.(88) of Ref. [5]) valid for $k_{2} \rightarrow 0$

$$
\frac{1}{\Omega(d)} \int \frac{d \Omega\left(d, \hat{k}_{2}\right)}{\left(\left(p-k_{2}\right)^{2}+1\right)^{N}} \approx\left(k_{2}^{2}\right)^{-\frac{N}{2}} \frac{\Gamma\left(\frac{d}{2}\right) \Gamma\left(\frac{N}{2}\right)}{2 \Gamma(N) \Gamma\left(\frac{1}{2}(d-N)\right)}, \quad k_{2} \rightarrow 0
$$

with $N=-(2 d-5)$, as in the term $\left(q^{2}+1\right)^{2 d-5}$ of Eq. (6.23), one obtains

$$
f_{4}\left(d, k_{2}^{2}\right)=a_{0}(d)\left[1+O\left(k_{2}^{2}\right)\right]+\frac{\Gamma\left(\frac{d}{2}\right) \Gamma\left(\frac{5}{2}-d\right)}{2 \Gamma(5-2 d) \Gamma\left(\frac{1}{2}(3 d-5)\right)} b_{0}(d)\left(k_{2}^{2}\right)^{\frac{1}{2}(2 d-5)}\left[1+O\left(k_{2}^{2}\right)\right] .
$$

Using the variable $1 /\left(k_{2}^{2}+1\right)=t$ in Eq.6.25) and inserting it in Eq.66.22) one gets the initial condition for $v_{4}(d, t)$ at the singular point $t=1$

$$
v_{4}(d, t)=\frac{a_{0}(d)}{\Gamma\left(\frac{d}{2}\right)}(1-t)^{\frac{d}{2}-1}[1+O(1-t)]+\frac{\Gamma\left(\frac{5}{2}-d\right)}{2 \Gamma(5-2 d) \Gamma\left(\frac{1}{2}(3 d-5)\right)} b_{0}(d)(1-t)^{\frac{1}{2}(3 d-7)}[1+O(1-t)] .
$$

By inspecting the equation (6.18) one gets that the behaviour at $t=0$ of $v_{4}(d, t)$ is

$$
v_{4}(d, t \rightarrow 0) \approx c_{4}^{(1)}(d) t^{-d+3}+c_{4}^{(2)}(d) t^{-2 d+5}+c_{4}^{(3)}(d) t^{\frac{1}{2}(-3 d+8)},
$$

so that for $d \rightarrow 4$ the integral (6.17) is convergent for $n \geq 4$.

All the quantities depending on $d$ are then systematically expanded in $d-4$, the series are truncated at some fixed number of terms, and the calculations with the truncated series are performed by using the program SYS [5]; as the first 12 terms of the series are lost in the intermediate steps of the calculations, in order to obtain the final results, from $1 / \epsilon^{4}$ up to $O\left(\epsilon^{6}\right), 23$ initial terms are needed. We solve finally the differential equation (6.18) with the initial condition (6.26) by a first expansions in series at $t=1$; due to the presence in Eq. (6.18) of a singular point at $t=-1 / 3$, to have fast convergence till $t=0$ we switch to subsequent series expansions at the intermediate points $1 / 2,1 / 4,1 / 8$ and 0 ; then we calculate the integral (6.17) for $n=4,5,6,7,8$ by integrating the series term by term (about 300 terms are needed to reach a precision of 77 digits). By applying repeatedly "top-down" the recurrence relation (6.16) to $I_{4}(d, 8), I_{4}(d, 7), I_{4}(d, 6)$, we obtain $I_{4}(d, 5)$ and $I_{4}(d, 4)$ (which are cross-checked with the values obtained by direct integration), then $I_{4}(d, 3), I_{4}(d, 2)$ and $I_{4}(d, 1)$

$$
\begin{aligned}
I_{4}(d, 1)= & \Gamma(1+\epsilon)^{3}\left[0.3333333333333333333333333333333 \epsilon^{-3}+\epsilon^{-2}\right. \\
& +1.3611111111111111111111111111111 \epsilon^{-1}+0.4254662447518595926183272929396 \\
& -31.041066530239171582155933528974 \epsilon-110.36756287612408836668594378878 \epsilon^{2} \\
& -611.01919192086881174365300012691 \epsilon^{3}-1734.0854215005636534710373849833 \epsilon^{4} \\
& \left.-7316.7112322583252172396619274906 \epsilon^{5}+O\left(\epsilon^{6}\right)\right] .
\end{aligned}
$$

Those values of $I_{4}(d, n)$ are used to determine the initial condition for $v_{5}(d, t)$, Eq.s(6.136.146.12). We then solve the differential equation (6.5) by expansions in series centered in the points $t=1,1 / 2,1 / 4$, $1 / 8,1 / 16$ and 0 (as above, this subdivision is due to the presence of a singular point at $t=-1 / 8$ ). By inspecting the equation (6.5) one gets that the behaviour at $t=0$ of $v_{4}(d, t)$ is

$$
v_{5}(d, t \rightarrow 0) \approx c_{5}^{(1)}(d) t^{-d+3}+c_{5}^{(2)}(d) t^{-2 d+5}+c_{5}^{(3)}(d) t^{(-3 d+8) / 2}+c_{5}^{(4)}(d) t^{(-5 d+12) / 2},
$$


so that for $d \rightarrow 4$ the integral (6.4) is surely convergent for $n \geq 5$; then we calculate the integral (6.4) for $n=5,6,7,8,9$ by integrating the series term by term. By using repeatedly "top-down" the recurrence relation (6.16) starting from $n=9$, we obtain $I_{5}(d, 6), I_{5}(d, 5)$ (used for cross-check), $I_{5}(d, 4), \ldots, I_{5}(d, 1)$. The result, up to the coefficient of order 5 in $(d-4)$ included, is

$$
\begin{aligned}
I_{5}(d, 1)= & \Gamma(1+\epsilon)^{4}\left[-0.25 \epsilon^{-4}-0.875 \epsilon^{-3}-1.416666666666666666666666666667 \epsilon^{-2}\right. \\
& -1.449652777777777777777777777778 \epsilon^{-1}-14.055442461941065705599451765901 \\
& -90.416062304531327135375791542063 \epsilon-1170.3684076603804614545918785105 \epsilon^{2} \\
& -5299.6462727245240600241060624284 \epsilon^{3}-37132.219579420859394978093377604 \epsilon^{4} \\
& \left.-144381.92488313453838475109116166 \epsilon^{5}+O\left(\epsilon^{6}\right)\right]
\end{aligned}
$$

Taking into account the normalization (6.2) one finds that the first 8 terms of Eq. (6.30) agree with Eq. (5.16).

We want only to mention that we have also independently checked the numerical result (6.30) by calculating the master integral with all masses equal to one by difference equations, and then by using the value so obtained as initial condition for the integration of a differential equation in the photon mass $\lambda$ from $\lambda=1$ to $\lambda=0$.

\section{The $x \rightarrow 0$ values.}

We evaluate in this section the $x \rightarrow 0$ values of $\Phi(d, x)$, Eq.(2.11).

By combining the familiar formulae

$$
\frac{1}{\left.\left.\left[(k-l)^{2}+a\right)\right]^{\alpha}\right]} \frac{1}{\left(l^{2}+b\right)^{\beta}}=\frac{\Gamma(\alpha+\beta)}{\Gamma(\alpha) \Gamma(\beta)} \int_{0}^{1} d x \frac{x^{\alpha-1}(1-x)^{\beta-1}}{\left[(l-x k)^{2}+x(1-x) k^{2}+a x+b(1-x)\right]^{\alpha+\beta}},
$$

and

$$
C^{-1}(d) \int \frac{d^{d} l}{(2 \pi)^{d-2}} \frac{1}{\left[(l-q)^{2}+c\right]^{n}}=\frac{1}{4} \frac{\Gamma\left(n-\frac{d}{2}\right)}{\Gamma\left(3-\frac{d}{2}\right) \Gamma(n)} \frac{1}{c^{n-\frac{d}{2}}},
$$

one gets

$$
C^{-1}(d) \int \frac{d^{d} l}{(2 \pi)^{d-2}} \frac{1}{\left[(k-l)^{2}+a\right]^{\alpha}} \frac{1}{\left(l^{2}+b\right)^{\beta}}=\frac{1}{4} \frac{\Gamma\left(\alpha+\beta-\frac{d}{2}\right)}{\Gamma(\alpha) \Gamma(\beta) \Gamma\left(3-\frac{d}{2}\right)} \int_{0}^{1} d x \frac{x^{\frac{d}{2}-\beta-1}(1-x)^{\frac{d}{2}-\alpha-1}}{\left[k^{2}+d(a, b, x)\right]^{\alpha+\beta-\frac{d}{2}}},
$$

where

$$
d(a, b, x)=\frac{a x+b(1-x)}{x(1-x)} .
$$

We rewrite Eq.(2.11) as

$$
\Phi(d, x)=\frac{C^{-4}(d)}{(2 \pi)^{4(d-2)}} \int \frac{d^{d} q}{\left[(p-q)^{2}+x^{2}\right]} \int \frac{d^{d} k_{2}}{\left(q-k_{2}\right)^{2}} \int \frac{d^{d} k_{1}}{\left(k_{2}-k_{1}\right)^{2}} \int \frac{d^{d} l}{\left[\left(k_{1}-l\right)^{2}+1\right]\left(l^{2}+1\right)},
$$

and then use repeatedly Eq.(7.3), using in the order the parameters $y, y_{1}, y_{2}, z$ for integrating the loops $l, k_{1}, k_{2}, q$, obtaining

$$
\begin{aligned}
\Phi(d, x) & =\frac{\Gamma(1-2(d-4))}{1024(d-3)(d-4)(2 d-5)(2 d-7) \Gamma^{4}\left(1-\frac{1}{2}(d-4)\right)} \\
& \times \int_{0}^{1} d y y^{\frac{d}{2}-2}(1-y)^{\frac{d}{2}-2} \int_{0}^{1} d y_{1} y_{1}^{\frac{d}{2}-2}\left(1-y_{1}\right)^{d-3} \int_{0}^{1} d y_{2} y_{2}^{\frac{d}{2}-2}\left(1-y_{2}\right)^{\frac{3}{2} d-4} \\
& \times \Psi\left(d, x, y, y_{1}, y_{2}\right),
\end{aligned}
$$


where

$$
\Psi\left(d, x, y, y_{1}, y_{2}\right)=\int_{0}^{1} d z \frac{z^{3-\frac{3}{2} d}}{\left[(1-z)^{2} x^{2}+z D\left(y, y_{1}, y_{2}\right)\right]^{5-2 d}}
$$

and

$$
D\left(y, y_{1}, y_{2}\right)=\frac{1}{y(1-y)\left(1-y_{1}\right)\left(1-y_{2}\right)} .
$$

The above formulae are valid for any $x$; form now on we take $0<x \ll 1$. For definiteness, we take also $d$ to be "just bigger" than 2 (i.e. $d=2+\eta$, with $0<\eta \ll 1$ ). The $z$-integral will be carried out first. To that aim, introduce an infinitesimal parameter $Z$, such that $0<x^{2} \ll Z \ll 1$ (a possible choice might be $Z=-x^{2} \ln x$, but the exact value of $Z$ will be irrelevant), and split the integration interval as

$$
\int_{0}^{1} d z=\int_{0}^{Z} d z+\int_{Z}^{1} d z
$$

correspondingly, we write the $z$-integral as

$$
\begin{aligned}
\Psi\left(d, x, y, y_{1}, y_{2}\right) & =\Psi_{1}\left(d, x, y, y_{1}, y_{2}\right)+\Psi_{2}\left(d, x, y, y_{1}, y_{2}\right), \\
\Psi_{1}\left(d, x, y, y_{1}, y_{2}\right) & =\int_{0}^{Z} d z \frac{z^{3-\frac{3}{2} d}}{\left[(1-z)^{2} x^{2}+z D\left(y, y_{1}, y_{2}\right)\right]^{5-2 d}}, \\
\Psi_{2}\left(d, x, y, y_{1}, y_{2}\right) & =\int_{Z}^{1} d z \frac{z^{3-\frac{3}{2} d}}{\left[(1-z)^{2} x^{2}+z D\left(y, y_{1}, y_{2}\right)\right]^{5-2 d}} .
\end{aligned}
$$

In the second term we can neglect $x^{2}$ in the denominator obtaining simply

$$
\begin{aligned}
\Psi_{2}\left(d, x, y, y_{1}, y_{2}\right) & \simeq \int_{Z}^{1} d z \frac{z^{3-\frac{3}{2} d}}{\left[z D\left(y, y_{1}, y_{2}\right)\right]^{5-2 d}}=D\left(y, y_{1}, y_{2}\right)^{2 d-5} \int_{Z}^{1} d z z^{\frac{d}{2}-2} \\
& =\frac{2}{d-2} D\left(y, y_{1}, y_{2}\right)^{2 d-5}
\end{aligned}
$$

where we have neglected the contribution from the lower integration limit $Z$, which is $Z^{\frac{d}{2}-1}$, as for $d$ just bigger than 2 it vanishes with $Z$.

The first term is slightly more delicate. To start with, as $0<z<Z \ll 1$ we can neglect $z$ with respect to 1 in $(1-z)^{2} x^{2}$

$$
\Psi_{1}\left(d, x, y, y_{1}, y_{2}\right) \simeq \int_{0}^{Z} d z \frac{z^{3-\frac{3}{2} d}}{\left[x^{2}+z D\left(y, y_{1}, y_{2}\right)\right]^{5-2 d}} ;
$$

for reasons which will be apparent in a moment, we rewrite it as

$$
\Psi_{1}\left(d, x, y, y_{1}, y_{2}\right)=\int_{0}^{Z} d z \frac{z^{\frac{d}{2}-2}}{\left[D\left(y, y_{1}, y_{2}\right)+\frac{x^{2}}{z}\right]^{5-2 d}}
$$

and integrate by parts the factor $z^{\frac{d}{2}-2}$; the result is

$$
\begin{aligned}
\Psi_{1}\left(d, x, y, y_{1}, y_{2}\right) & =\frac{2}{d-2}\left\{\left.\frac{z^{4-\frac{3}{2} d}}{\left[x^{2}+z D\left(y, y_{1}, y_{2}\right)\right]^{5-2 d}}\right|_{0} ^{Z}+(2 d-5) x^{2} \int_{0}^{Z} d z \frac{z^{3-\frac{3}{2} d}}{\left[x^{2}+z D\left(y, y_{1}, y_{2}\right)\right]^{6-2 d}} \cdot\right\} \\
& =2 \frac{2 d-5}{d-2} x^{2} \int_{0}^{Z} d z \frac{z^{3-\frac{3}{2} d}}{\left[x^{2}+z D\left(y, y_{1}, y_{2}\right)\right]^{6-2 d}},
\end{aligned}
$$


as the end-point contributions vanish for $d$ just bigger than 2. We can now modify the integration interval for $\Psi_{1}\left(d, x, y, y_{1}, y_{2}\right)$ into

$$
\int_{0}^{Z} d z=\int_{0}^{\infty} d z-\int_{Z}^{\infty} d z
$$

and write correspondingly

$$
\Psi_{1}\left(d, x, y, y_{1}, y_{2}\right)=\Psi_{1 \infty}\left(d, x, y, y_{1}, y_{2}\right)-\Psi_{1 Z}\left(d, x, y, y_{1}, y_{2}\right),
$$

where, thanks to the previous integration by parts, the two resulting $z$-integrals (from 0 to $\infty$ for the first, from $Z$ to $\infty$ for the second) are now both convergent for $d$ just bigger than 2 .

We start again from the second term,

$$
\Psi_{1 Z}\left(d, x, y, y_{1}, y_{2}\right)=2 \frac{2 d-5}{d-2} x^{2} \int_{Z}^{\infty} d z \frac{z^{3-\frac{3}{2} d}}{\left[x^{2}+z D\left(y, y_{1}, y_{2}\right)\right]^{6-2 d}} ;
$$

in the denominator we can neglect $x^{2}$, the resulting integral is trivial and the result can be written as

$$
\Psi_{1 Z}\left(d, x, y, y_{1}, y_{2}\right)=2 \frac{2 d-5}{d-2} \frac{1}{\left[D\left(y, y_{1}, y_{2}\right)\right]^{6-2 d}} \frac{2}{d-4}\left\{\left(\frac{x^{2}}{z}\right) z^{\frac{d}{2}-1}\right\}_{Z}^{\infty}
$$

which vanishes with $Z$ (recall $x^{2} \ll Z$ ), so that

$$
\Psi_{1 Z}\left(d, x, y, y_{1}, y_{2}\right)=0 \text {. }
$$

In the first term of Eq. (17.10), $\Psi_{1 \infty}\left(d, x, y, y_{1}, y_{2}\right)$, we substitute $z=t x^{2} / D\left(y, y_{1}, y_{2}\right)$, obtaining

$$
\Psi_{1 \infty}\left(d, x, y, y_{1}, y_{2}\right)=2 \frac{2 d-5}{d-2} x^{(d-2)}\left[D\left(y, y_{1}, y_{2}\right)\right]^{\frac{3}{2} d-4} \int_{0}^{\infty} d t \frac{t^{3-\frac{3}{2} d}}{(1+t)^{6-2 d}} .
$$

By using the second representation of Euler's Beta function

$$
\begin{aligned}
B(\alpha, \beta) & =\frac{\Gamma(\alpha) \Gamma(\beta)}{\Gamma(\alpha+\beta)} \\
& =\int_{0}^{1} d u u^{\alpha-1}(1-u)^{\beta-1}=\int_{0}^{\infty} d t \frac{t^{\alpha-1}}{(1+t)^{\alpha+\beta}}
\end{aligned}
$$

we finally obtain

$$
\Psi_{1 \infty}\left(d, x, y, y_{1}, y_{2}\right)=2 \frac{2 d-5}{d-2} B\left(4-\frac{3}{2} d, 2-\frac{1}{2} d\right) x^{(d-2)}\left[D\left(y, y_{1}, y_{2}\right)\right]^{\frac{3}{2} d-4} .
$$

By collecting the results Eq.s (7.1377.11 7.10 7.977.8), one obtains the value of the function $\Psi\left(d, x, y, y_{1}, y_{2}\right)$ to be substituted in Eq.(17.5); recalling Eq.(7.7) one sees that all the remaining integrations factorize and can be carried out in terms of Euler's Beta-functions Eq. (7.12).

It is clear that the final result for Eq.(2.11) for $x \rightarrow 0$ consists of two terms, the first constant (independent of $x$ ), the second proportional to $x^{d-2}$. In the notation of Eq.(3.5) we have

$$
\begin{aligned}
A_{0}^{(1)} & =-\frac{3 d-11}{8(d-2)(d-3)(d-4)^{3}(2 d-5)(2 d-7)(3 d-8)(3 d-10)} \\
& \times \frac{\Gamma(1-(d-4)) \Gamma(1-2(d-4)) \Gamma^{2}\left(1+\frac{1}{2}(d-4)\right) \Gamma^{2}\left(1-\frac{3}{2}(d-4)\right)}{\Gamma^{4}\left(1-\frac{1}{2}(d-4)\right) \Gamma(1-3(d-4))} \\
A_{0}^{(2)} & =-\frac{2(2 d-7)}{3(d-2)^{2}(d-3)(d-4)^{4}(3 d-8)(3 d-10)} \\
& \times \frac{\Gamma\left(1+\frac{1}{2}(d-4)\right) \Gamma\left(1-\frac{3}{2}(d-4)\right) \Gamma^{2}(1-(d-4))}{\Gamma^{2}\left(1-\frac{1}{2}(d-4)\right) \Gamma(1-2(d-4))} .
\end{aligned}
$$




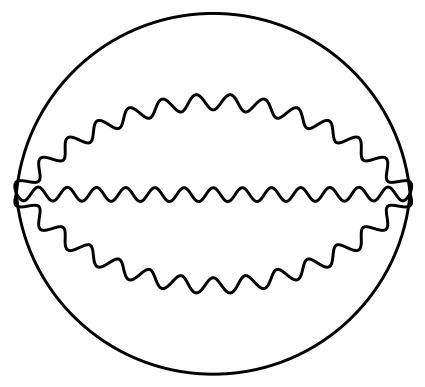

Figure 3: The 4-loop watermelon graph.

Let us observe that the term $A_{0}^{(1)}$ is the value of the vacuum graph in Fig.3., in agreement with the result in Eq.(A.12) of [13] (up to a different normalization).

\section{Acknowledgments}

We are grateful to J. Vermaseren for his kind assistance in the use of the algebra manipulating program FORM [9], by which all our calculations were carried out.

\section{References}

[1] A. V. Kotikov, Phys. Lett. B 254 (1991) 158.

[2] E. Remiddi, Nuovo Cim. A 110 (1997) 1435 arXiv:hep-th/9711188.

[3] M. Caffo, H. Czyz, S. Laporta and E. Remiddi, Nuovo Cim. A 111 (1998) 365 arXiv:hep-th/9805118.

[4] T. Gehrmann and E. Remiddi, Nucl. Phys. B 580 (2000) 485 arXiv:hep-ph/9912329.

[5] S. Laporta, Int. J. Mod. Phys. A 15 (2000), 5087 hep-ph/0102033.

[6] S. Laporta, Phys. Lett. B 523 (2001), 95 hep-ph/0111123.

[7] M. Argeri, P. Mastrolia, E. Remiddi, Nucl. Phys. B 631 (2002), 388 hep-ph/0202123.

[8] P. Mastrolia and E. Remiddi, Nucl. Phys. B 657 (2003), 397 hep-ph/0211451.

[9] J.A.M. Vermaseren, Symbolic Manipulation with FORM, Version 2, CAN, Amsterdam, 1991; New features of FORM, math-ph/0010025.

[10] E. Remiddi, J.A.M. Vermaseren, Int. J. Mod. Phys. A 15 (2002), 725 hep-ph/9905237.

[11] V. A. Smirnov, Applied Asymptotic Expansions in Momenta and Masses, Springer tracts in modern physics 177, Springer(2002) Berlin, Germany.

[12] J. Vermaseren, table available from the URL http://www.nikhef.nl/ form/FORMdistribution/packages/harmpol/index.htlm

[13] K. Kajantie, M. Laine, K. Rummukainen, and Y. Schroder, JHEP 04 (2003), 036, hep-ph/0304048. 